\title{
Comparative profiling of biofilm-production, quorum sensing system and virulence genes in human and ovine non-aureus staphylococci
}

\section{Elisa Azara}

Istituto Zooprofilattico Sperimentale della Sardegna G Pegreffi

\section{Carla Maria Longheu}

Istituto Zooprofilattico Sperimentale della Sardegna G Pegreffi

\section{Sonia Attene}

Ospedale San Francesco

\section{Silvana Sanna}

Azienda Ospedaliera Universitaria Sassari

\section{Marco Sale}

Ospedale Antonio Segni

\section{Maria Filippa Addis}

Università degli Studi di Milano Dipartimento di Medicina Veterinaria: Universita degli Studi di Milano Dipartimento di Medicina Veterinaria

Sebastiana Tola ( $\square$ sebastiana.tola@izs-sardegna.it)

Istituto Zooprofilattico Sperimentale della Sardegna G Pegreffi https://orcid.org/0000-0002-8488-3773

\section{Research article}

Keywords: on-aureus staphylococci, human, ovine, biofilm, adhesins, toxins, quorum sensing

Posted Date: February 26th, 2021

DOI: https://doi.org/10.21203/rs.3.rs-272051/v1

License: (c) (1) This work is licensed under a Creative Commons Attribution 4.0 International License. Read Full License 


\section{Abstract}

Background: This study assessed the genetic characteristics shared by non-aureus staphylococci (NAS) responsible for human infections and those causing mastitis in dairy ewes. In a collaboration between animal and human health care professionals, we collected and identified 125 ovine and 70 human NAS isolates and compared them for biofilm production, presence of autolysins, microbial surface components recognizing adhesive matrix molecules (MSCRAMMS), pyrogenic toxins, and agr alleles regulating quorum-sensing systems. Ovine NAS included: S. epidermidis (57), S. chromogenes (29), S. haemolyticus (17), S. simulans (8), S. caprae (6), S. warneri (5), S. saprophyticus, S. intermedius, and S. muscae (1 each) while human NAS included: S. haemolyticus (28), S. epidermidis (26), S. hominis (4), $S$. lugdunensis (4), S. capitis (3), S. warneri (2), S. xylosus, S. pasteuri, and S. saprophyticus subsp. bovis (1 each).

Results: Based on colony characteristics on Congo Red Agar, 4 (3.2\%) ovine, and 49 (70\%) human isolates produced biofilm. Few $S$. epidermidis isolates harbored the icaA/D genes coding for the polysaccharide intercellular adhesin (PIA) and the bhp, aap, and embp genes coding biofilm accumulation proteins. PCR amplification of the genes coding for autolysins (at/E and aae), microbial surface components recognizing adhesive matrix molecules (MSCRAMMs, $s d r G$ and $s d r F$ ), enterotoxins (sea, seb, sec, sed, and see), and the toxic shock syndrome toxin (tsst), revealed that $40 \%, 39.2 \%, 47.2 \%$ and $52.8 \%$ of the sheep isolates carried at $/ E$, aae, sdrF and $s d r G$, respectively, against $37.1 \%, 42.8 \%$, $32.8 \%$, and $60 \%$ of human isolates. Enterotoxins and tsst were not detected. Fifty-nine sheep isolates (all S. epidermidis, 1 S. chromogenes, and 1 S. haemolyticus) and 27 human NAS (all S. epidermidis and 1 S. warneri) were positive for the accessory gene regulator (agr), responsible for the regulation of virulence factors: agr-3se (57.8\%) followed by agr-1 se (36.8\%) predominated in sheep, while agr-1 se $(65.4 \%)$, followed by agr-2se (34.6\%) predominated in humans.

Conclusions: This comparative study provided a detailed characterization of the putative virulence genes present in human and ovine NAS and indicated that the ability to form biofilms, observed mainly in human S. epidermidis, could be a major virulence factor facilitating colonization, infection, diffusion, and resistance.

\section{Background}

Half of the total Italian dairy sheep stock is farmed in Sardinia, an island located in the Mediterranean Sea. Sardinia has approximately 3.5 million dairy sheep, while the population is around 1.6 million inhabitants. Accordingly, a relevant part of the regional economy relies on dairy sheep farming, and the control of intra-mammary infections (IMI) is consequently of the greatest importance.

Several reports indicate that non-aureus staphylococci (NAS) are the most prevalent bacteria causing subclinical mastitis in sheep and goats [1-4], creating opportunities for the exchange of colonizing as well as pathogenic microorganisms among sheep and farmers, with their antimicrobial-resistance and 
pathogenicity gene pools. Of note, NAS have emerged as predominant nosocomial agents capable of causing infection in debilitated or compromised patients and are associated with catheter-related and other indwelling medical device-related infections [5]. NAS, and in particular S. epidermidis, can produce a multicellular biofilm that decreases the antibiotic concentration within the colony and promotes multiplication and enhances survival of invading bacteria [6].

The four main biofilm formation steps are adherence/aggregation, accumulation, maturation, and detachment [7]. Specific staphylococcal autolysins, including AtIE and Aae [8, 9], mediate initial adhesion to surfaces, and microbial surface components recognizing adhesive matrix molecules (MSCRAMMs) further promote colonization and serum protein binding [10]. The best known S. epidermidis MSCRAMMs are the fibrinogen-binding protein SdrG [11], and the collagen/keratin-binding protein SdrF [12, 13]. The extracellular polysaccharide intercellular adhesin (PIA) mediates the accumulation phase, and in $S$. epidermidis this step is mediated by icaABCD-encoded proteins [6]. However, PIA-independent biofilms involving a accumulation-âssociated protein (Aap), biofilm homologue protein (Bhp) and extracellular matrix-binding protein (Embp) $[14,15]$ have also been reported.

The production of various toxins can contribute to NAS virulence [16], including staphylococcal enterotoxins (SES) and toxic shock syndrome toxin 1 (TSST-1) [17]. Five serological types of SEs are typically known (SEA to SEE), but new types of SEs (SEG to SE1V) have also been identified and characterized $[18,19]$. The quorum-sensing system (QS) agr, i.e. accessory gene regulator $[20,21]$ regulates biofilm formation, intercellular communication, and numerous virulence factors including toxins and autolysins. Three distinct genetic groups (types 1,2, and 3 ) based on the agr locus polymorphism have been described in S. epidermidis [22], but data on the genetic polymorphisms of the agr locus in different species of NAS were not available in the scientific literature at the beginning of this investigation.

We compared the molecular characteristics of NAS isolated from ovine mastitis and human clinical specimens with the following aims: 1) assess the biofilm production characteristics by phenotypic and genotypic methods, 2) carry out genotypic screening for a set of MSCRAMMs, autolysins, enterotoxins and tsst-1 genes and 3) investigate the agr locus and its genetic polymorphism.

\section{Results}

\section{Ovine NAS: general findings}

We analyzed a total of 125 isolates, including $S$. epidermidis $(n=57), S$. chromogenes $(n=29), S$. haemolyticus $(\mathrm{n}=17)$, S. simulans $(\mathrm{n}=8)$, S. caprae $(\mathrm{n}=6), S$. warneri $(\mathrm{n}=5)$, S. saprophyticus $(\mathrm{n}=1), S$. intermedius $(n=1)$ and $S$. muscae $(n=1)$. Table 1 reports the isolates included in the study, while Table 2 reports the primers used for PCR amplifications.

Table 3 summarizes biofilm formation results, evaluated by the phenotypic characteristics of bacterial colonies grown on Congo Red Agar (CRA) and by PCR detection of icaA/D, bhp, aap, embp, at/E, aae, sdrF, 
sdrG and agr. Out of 125 isolates examined, only 4 (3.2\%) were classified as strong biofilm producers because their colonies exhibited a very black color; the remaining $121(96.8 \%)$ as non-biofilm producers due to bordeaux or red color. One isolate harbored both icaA and icaD genes while two had only the icaA gene: one of them was considered as non-biofilm-producing phenotype. On the other hand, $37(29.6 \%), 22$ (17.6\%) and $63(50.4 \%)$ isolates possessed the bhp, aap and embp genes, respectively (Table 3 ). Concerning autolysin genes, 50 (40\%) isolates were PCR positive for altE while 49 (39.2\%) were positive for aae. Concerning adhesion (MSCRAMMS) factors, 59 (47.2\%) and $66(52.8 \%)$ isolates harbored sdrF and $s d r G$ genes, respectively. Concerning the agrtype, $21(16.8 \%)$ isolates belonged to agr-1 whilst 33 (26.4\%) to agr-3. None of the isolates belonged to type 2 . The toxin genes analyzed did not produce amplification (data not shown).

\section{Ovine S. epidermidis}

S. epidermidis was the most represented ovine NAS. Out of 57 isolates, $2(3.5 \%)$ were strong biofilm producers but only one harbored both icaA/D genes. On the other hand, $54(94.7 \%), 30(52.6 \%)$ and 20 $(16 \%)$ isolates possessed embp, bhp and aap genes, respectively. Concerning autolysin genes, 48 (84.2\%) were PCR-positive for at/E and 46 (80.7\%) for aae. High percentages were found for MSCRAMMS genes: $87.7 \%$ for $s d f G$ and $78.9 \%$ for $s d r F$. All S. epidermidis isolates were positive for the agr locus: 33 (57.8\%) belonged to agr $3_{\text {se }}$ while $21(36.8 \%)$ to agr- $1_{\text {se }}$ (Table 3$)$. Three isolates were non-typeable.

\section{S. chromogenes, S. haemolyticus and minor ovine NAS}

S. chromogenes $(\mathrm{n}=29)$ and $S$. haemolyticus $(\mathrm{n}=17)$ were the most prevalent species in ovine milk samples after $S$. epidermidis. Table 3 shows that almost all isolates were non-biofilm producers, except one S. caprae and one S. muscae.

\section{Human NAS: general findings}

Table 3 summarizes the distribution of virulence genes in the 70 human NAS, including S. haemolyticus, S. epidermidis, S. lugdunensis, S. hominis, S. capitis, S. warneri, S. xylosus, S. pasteuri and S. saprophyticus subsp. bovis.

\section{Human S. haemolyticus}

Out of 28 S. haemolyticus isolates examined, 16 (57.1\%) were classified as strong biofilm producers by CRA because their colonies exhibited a very black color; the remaining 12 as non-biofilm producers due to bordeaux or red color. None of $S$. haemolyticus isolates harbored icaA/D, bhp and embp. On the contrary, three black (2 from blood and 1 from nasal swab) and one bordeaux (from glans swab) isolates possessed the aap gene. No amplification was observed for at|E, aae, sdrF, sdrG genes and agr locus (Table 3) and for all toxin genes (data not shown).

\section{Human S. epidermidis}


Among the 26 S. epidermidis isolates, 22 (84.6\%) showed a very black color; ten (38.5\%) of them (3 from catheter, 3 from blood, 2 from nasal swab, 1 from skin swab and 1 from pus) harbored both icaA and icaD. The remaining isolates were icaA/D negative. However, 16 (61.5\%), 25 (96.1\%) and 23 (88.5\%) isolates possessed the bhp, aap and embp genes, respectively (Table 3). Out of 10 icaA/D positive isolates, 5 were also positive for the other biofilm genes analyzed. Among the 4 non-biofilm-producing phenotypes, all were icaA/D negative but 3 ( 2 from pus and 1 from oral swab) harbored aap and embp while 1 (from skin swab) possessed all bhp, aap and embp genes. Data on the prevalence of autolysins and MSCRAMMs genes by PCR are shown in Table 3. Concerning autolysin genes, all isolates were PCR positive for aae and almost all $(25 / 26=96.1 \%)$ were positive for altE. Regarding adhesion factors, all $S$. epidermidis isolates were positive for sdrG and 21/26 (80.8\%) harbored sdrF. In four of them (2 from pus, 1 from biopsy and 1 from skin swab), a PCR product smaller than the expected size was observed. Sequence analysis of these amplicons showed the absence of an $84 \mathrm{bp}$ fragment. Determination of the agrtype was performed in all S. epidermidis isolates: 17 (65.4\%) belonged to agr-1 se whilst 9 (34.6\%) to agr-2 ${ }_{\text {se }}$ (Table 4). Among the 17 agr-1 se isolates, only 3 ( 1 from femoral venous catheter, 1 from nasal swab and 1 from pus) carried simultaneously icaA/D, bhp, aap, embp, at/E, aae, sdrF and sdrG genes associated with biofilm formation. Among the 9 agr- $2_{\text {se }}$ isolates, only 1 (from blood) possessed these genes. Regarding the pyrogenic toxin genes, amplification was not observed in any of the S. epidermidis isolates or in the remaining staphylococci (data not shown).

\section{Minor human NAS}

Out of the 4 S. Iugdunensis isolates examined, 3 (2 from peritoneal fluid and 1 from vaginal swab) displayed a very black colour on CRA. However, none of them harbored neither icaA/D nor any other gene examined, except the sdrG gene.

Negative results were also obtained for all $S$. hominis isolates, except for a PCR positivity for aap and sdrG genes. Among the $3 \mathrm{~S}$. capitis isolates, 1 (from skin swab) exhibited black color whereas 2 were phenotypically red but, despite this, all isolates were PCR-positive for icaA and sdrG genes. Table 4 reports the PCR results for S. warneri, S. xylosus, S. pasteuri, and S. saprophyticus subsp. bovis. Of note, one bordeaux S. warneri isolate (from fluid drainage) was PCR negative for icaA/D and bhp genes but positive for aap, embp, at/E, aae, sdrG/F and agrgenes. However, we were not able to type the agr locus.

\section{Discussion}

This work results from an integrated collaboration between animal and human health care professionals for understanding if non-aureus staphylococci (NAS) responsible for human diseases share some genetic characteristics with those circulating in sheep. A total of 195 NAS isolates, 125 from ovine mastitis and 70 from human clinical specimens, were analyzed for biofilm production and presence of autolysins, MSCRAMMS and pyrogenic toxins. We also typed agr alleles by PCR because many virulence determinants involved in staphylococcal infections, including autolysins, adhesins and toxins are regulated by quorum sensing system [16]. In sheep, the main NAS detected were S. epidermidis followed 
by S. chromogenes and S. haemolyticus. In humans, we found primarily S. haemolyticus and $S$. epidermidis, followed by S. Iugdunensis, S. hominis, S. capitis, S. warneri, S. xylosus, S. pasteuri and S. saprophyticus subsp. bovis. S. haemolyticus has been associated with septicemia in neonates and skin infections; $S$. epidermidis is the main pathogen isolated in catheter-associated bloodstream infections (BSI); S. Iugdunensis can cause acute endocarditis; S. hominis and S. capitis may induce BSI in neonates; $S$. warneri is associated with device-related bone and joint infections, while S. pasteuri, $S$. xylosus and $S$. saprophyticus subsp. bovis are not associated to a particular clinical infection, and their appearance as nosocomial pathogens could be related to previous contact with animals, mainly pig, cattle, sheep and goats [23]; $S$. epidermidis represents the most frequently isolated species from ovine mastitis and human clinical specimens $[1,23,24]$.

Overall, 53 NAS were able to form biofilm onto CRA plates, according to the Arciola criteria [25]; however, the percentage of strong biofilm producers in sheep isolates was much lower than for human isolates: $3.2 \%(4 / 125)$ versus 70\% (49/70). Only in $11 \mathrm{~S}$. epidermidis isolates (10 human and 1 ovine), we found a correlation between black color development and ica operon presence. Some authors proposed to use this correlation as a pathogenesis marker to distinguish invasive from commensal isolates [26, 27]. However, we and others $[15,28,29]$ demonstrated that PCR positivity for icaA/icaD genes can also be found in non-biofilm producers. Since the correlation between biofilm production and positivity for ica, bhp, aap and embp genes is not well known; we suggest considering all isolates that possess such genes as potentially invasive. In this work, only $1 \mathrm{~S}$. epidermidis with these characteristics was isolated from ovine mastitis while the other 5 derived from femoral venous catheter, blood, nasal swab, skin swab and pus. Nasal and skin swabs were collected from patients in the intensive care unit. Noteworthy, high positivity (80.7 to 100 percent) for the genes encoding the bifunctional adhesins/autolysins AtIE and Aae was found in both animal and human $S$. epidermidis isolates $[3,5]$. In addition to bacteriolytic activity, AtIE and Aae act as adhesins by binding noncovalently to vitronectin and by causing the release of extracellular DNA (eDNA), a critical adherence/aggregation factor in biofilm formation [30]. The presence of the at $/ E$ and aae genes was accompanied by a high prevalence of embp, sdrG and $s d r F$, encoding proteins that mediate adherence to substrates containing fibronectin, fibrinogen and collagen $[4,31]$. This observation confirms a pivotal role of AtIE, Aae, Embp, and SdrG in the S. epidermidis pathogenesis.

The agr locus is a regulatory system that responds to host and environmental stimuli and controls the production of many virulence factors [21]. In S. epidermidis, three distinct agr groups have been recognized [22]. Li et al. [32] have linked genetic polymorphism of the agrlocus to pathogenicity; group$1_{\text {se }}$ was associated with pathogenicity, while healthy people mainly carried group-2se. In our human $S$. epidermidis isolates, agr $1_{\text {se }}$ was predominant $(n=17)$, followed by agr $2_{s e}(n=9)$. It is interesting to notice that almost all $(\mathrm{n}=9)$ isolates possessing ica genes belonged to agr $1_{\mathrm{se}}$. This may suggest a correlation of these virulence genes with a specific agr locus. However, other $8 i c a A^{-} / D^{-}$isolates were present in the group- $1_{\text {se. }}$. The feature shared by all 17 isolates belonging to this group was the PCR positivity for the at $\mathbb{E}$, aae and $s d r G$ genes. On the other hand, among the 9 isolates grouped in the agr $-2 \mathrm{se}_{1} 1$ (from blood) was $i c a A^{+} / D^{+}$, while the remaining ones were ica-negative. The common denominator of these 9 isolates were 
the PCR positivity for the aap, aae, sdrG and embp genes. These findings suggest that the relationship between agrgroups and pathogenicity of $S$. epidermidis will require further investigation. As observed in our previous study [28], agr-3 ${ }_{\text {se }}(\mathrm{n}=33)$ was predominant among ovine $S$. epidermidis isolates followed by agr-1 $1_{\text {se }}(\mathrm{n}=21)$. These results may suggest a possible transmission of $S$. epidermidis isolates from the milkers to the ewes.

Unlike $S$. haemolyticus from ovine mastitis, several human isolates exhibited a positive result in the qualitative test CRA but did not harbor icaA/D. According to Fredheim et al. [33], S. haemolyticus mainly produces a PIA-independent biofilm. However, in the present study, we did not detect the bhp and embp genes in any of the investigated isolates by PCR. Only 4 human $S$. haemolyticus ( 3 black and 1 bordeaux isolates) possessed the aap gene coding a protein that mediates biofilm formation in strains lacking the ica genes [11]. Our data suggest that ica, bhp, and embp genes do not contribute significantly to $\mathrm{S}$. haemolyticus biofilms' protein components.

In $S$. aureus and in many other bacteria, toxins are critical contributors to aggressive virulence, even though S. epidermidis is not generally accepted as an enterotoxin producer [34, 35]. Based on our findings, the primary enterotoxin genes (sea, seb, sec, sed and see) and the tsst-1 gene were absent in all ovine and human NAS analyzed. On the contrary, Pedroso et al. [16] and Da Cunha et al. [36] detected high percentage of sea and sec genes in coagulase-negative staphylococci collected from hospitals of Brazil; also, Giormezis et al. [35] found a higher number of isolates positive for tsst among NAS collected from hospitals in Greece.

\section{Conclusion}

In conclusion, our study demonstrated the presence of intercellular adhesion genes (icaAB) and other genes related to biofilm formation only in $S$. epidermidis. At the same time, $S$. caprae and $S$. saprophyticus (ovine NAS) and S. capitis and S. xylosus (human NAS) harbored only icaA. The remaining isolates carried few virulence determinants. The biofilm formation ability observed mainly in human isolates, especially $S$. epidermidis, could be a significant virulence factor facilitating colonization, infection, diffusion, and resistance.

\section{Methods}

\section{Isolate collection}

Ovine isolates: A total of 125 NAS isolates were collected from sheep milk samples in different provinces of Sardinia (Italy) over a period of 9 months (April-December 2017) (Table 1). Isolates were identified as: S. epidermidis $(\mathrm{n}=57)$, S. chromogenes $(\mathrm{n}=29)$, S. haemolyticus $(\mathrm{n}=17)$, S. simulans $(\mathrm{n}=8)$, S. caprae $(\mathrm{n}=6)$, S. warneri $(\mathrm{n}=5), S$. saprophyticus $(\mathrm{n}=1), S$. intermedius $(\mathrm{n}=1)$, and $S$. muscae $(\mathrm{n}=1)$, by means of PCRRFLP [24]. 
Human isolates: During the same period, 70 NAS isolates were collected from different clinical specimens at the microbiology laboratories of three major Sardinia hospitals. Isolates and their origin are summarized in Table 1; $90 \%$ of the human NAS were recovered from hospitalized patients in intensive care unit, hematology, and orthopedics. The 70 isolates were identified by PCR-RFLP as S. haemolyticus $(\mathrm{n}=28), S$. epidermidis $(\mathrm{n}=26)$, S. Iugdunensis $(\mathrm{n}=4), S$. hominis $(\mathrm{n}=4), S$. capitis $(\mathrm{n}=3), S$. warneri $(\mathrm{n}=2), S$. $\operatorname{xylosus}(\mathrm{n}=1)$, S. pasteuri $(\mathrm{n}=1)$, and S. saprophyticus subsp. bovis $(\mathrm{n}=1)$ [24].

Statements of owner consent or patient consent were not required in this case since personal or sensitive data never accompanied samples. All Isolates were anonymized regarding the originating animal, flock, or patient, and were processed for phenotypic and molecular analyses without any original information linked to them.

\section{Phenotyping evaluation of biofilm production}

Phenotypic evaluation of biofilm production was assessed on CRA [37]. On this medium, slime-producing strains form very black, black and almost black colonies, while non-producing strains develop bordeaux, red and very red colonies [25]. CRA plates were prepared and incubated as described by Abbondio et al. [28]. We used the $S$. epidermidis reference strain (RS) RP62A (ATCC 35984) as a positive control and ATCC 12228 as a negative control.

\section{Detection of biofilm, autolysins, MSCRAMMs and pyrogenic toxins genes}

Genomic DNA was extracted from all 195 NAS isolates and RS according to Onni et al. [38]. Primer sets for detecting of genes related to biofilm production, autolysins, MSCRAMMs and pyrogenic toxins are detailed in Table 2. PCR tests were carried out in a GeneAmp9700 DNA thermal cycler (Applied Biosystems, now Thermo Fisher Scientific, Waltham, MA, USA). The following RS were used as positive controls: S. epidermidis ATCC 35984 (ica), S. epidermidis ATCC 35984 (at/E, aae, sdrF, sdrG), S. aureus ATCC 13565 (sea), S. aureus ATCC 14458 (seb), S. aureus ATCC19095 (sec), S. aureus ATCC 23235 (sed), S. aureus ATCC 27664 (see) and S. aureus ATCC 33586 (tsst-1).

\section{Typing of agr alleles}

A 200 bp conserved region of the agroperon was amplified as described previously [16]. For isolate

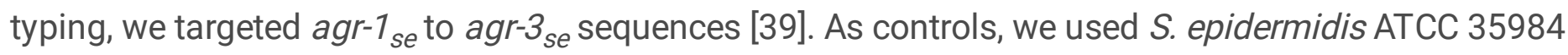
$\left(\right.$ agr-1 $\left.{ }_{\text {se }}\right)$, S. epidermidis isolate 1037 (agr-2s $\left.s_{e}\right)$ and S. epidermidis isolate 43027 (agr-3 $3_{\text {se) }}$. Table 2 reports primer sets and related references.

\section{Abbreviations}

CRA, Congo Red Agar; MSCRAMMs, microbial surface components recognizing adhesive matrix molecules; NAS, non-aureus staphylococci; QS, quorum-sensing system. 


\section{Declarations}

\section{Ethics approval and consent to participate}

Does not apply.

\section{Consent for publication}

Not applicable

\section{Availability of data and material}

The data used in this study is under the regulation of the Istituto Zooprofilattico Sperimentale della Sardegna. It will be available from the corresponding author.

\section{Competing interests}

The authors declare that they have no conflicts of interest associated with this study.

\section{Funding}

This work was supported by Istituto Zooprofilattico Sperimentale della Sardegna, Italy. The funding body have no role in the design of the study, writing the manuscript and the collection, analysis and interpretation of data.

\section{Authors' contributions}

EA: carried out isolation and identification of ovine NAS; performed the experiments; analyzed the data. CML: carried out isolation and identification of ovine NAS; performed the experiments; SA, SS, and MS: carried out isolation and identification of human NAS; MFA: interpreted the data; drafted and revised the manuscript; ST: Conceived the study, analyzed, and interpreted the data; drafted the manuscript; supervised the project. All authors read, edited, and approved the final version of the manuscript.

\section{Acknowledgements}

Not applicable.

\section{References}

1. Marogna G, Rolesu S, Lollai S, Tola S, Leori G. Clinical findings in sheep farms affected by recurrent bacterial mastitis. Small Rumin Res. 2010;88:119-25. doi:10.1016/j.smallrumres.2009.12.019.

2. Onni T, Vidili A, Bandino E, Marogna G, Schianchi S, Tola S. Identification of coagulase-negative staphylococci isolated from caprine milk samples by PCR-RFLP of groEL gene. Small Rumin Res. 2012;104:185-90. doi:10.1016/j.smallrumres.2011.10.004. 
3. Martins KB, Faccioli PY, Bonesso MF, Fernandes S, Oliveira AA, Dantas A, et al. Characteristics of resistance and virulence factors in different species of coagulase-negative staphylococci isolated from milk of healthy sheep and animals with subclinical mastitis. J Dairy Sci. 2017;100:2184-95. doi:10.3168/jds.2016-11583.

4. Vanderhaeghen W, Piepers S, Leroy F, Van Coillie E, Haesebrouck F, De Vliegher S. Identification, typing, ecology and epidemiology of coagulase negative staphylococci associated with ruminants. Vet J. 2015;203:44-51. doi:10.1016/j.tvjl.2014.11.001.

5. Von Eiff C, Peters G, Heilmann C. Pathogenesis of infections due to coagulase-negative staphylococci. Lancet Infectious Diseases. 2002;2:677-85. doi:10.1016/S1473-3099(02)00438-3.

6. Mack D, Davies AP, Harris LG, Rohde H, Horstkotte MA, Knobloch JK-M. Microbial interactions in Staphylococcus epidermidis biofilms. Analytical and Bioanalytical Chemistry. 2007;387:399-408. doi:10.1007/s00216-006-0745-2.

7. Fey PD, Olson ME. Current concepts in biofilm formation of Staphylococcus epidermidis. Future Microbiology. 2010;5:917-33. doi:10.2217/fmb.10.56.

8. Heilmann C, Hussain M, Peters G, Götz F. Evidence for autolysin-mediated primary attachment of Staphylococcus epidermidis to a polystyrene surface. Mol Microbiol. 1997;24:1013-24. doi:10.1046/j.1365-2958.1997.4101774.x.

9. Heilmann C, Thumm G, Chhatwal GS, Hartleib J, Uekötter A, Peters G. Identification and characterization of a novel autolysin (Aae) with adhesive properties from Staphylococcus epidermidis. Microbiology. 2003;149:2769-78. doi:10.1099/mic.0.26527-0.

10. Otto M. Molecular basis of Staphylococcus epidermidis infections. Seminars in Immunopathology. 2012;34:201-14. doi:10.1007/s00281-011-0296-2.

11. Pei L, Flock JI. Lack of fbe, the gene for a fibrinogen-binding protein from Staphylococcus epidermidis, reduces its adherence to fibrinogen coated surfaces. Microb Pathog. 2001;31:185-93. doi:10.1006/mpat.2001.0462.

12. Arrecubieta $C$, Toba FA, Von Bayern M, Akashi H, Deng MC, Naka Y, et al. SdrF, a Staphylococcus epidermidis surface protein, contributes to the initiation of ventricular assist device driveline-related infections. PLoS Pathog. 2009;5:e1000411. doi:10.1371/journal.ppat.1000411.

13. Trivedi S, Uhlemann AC, Herman-Bausier P, Sullivan SB, Sowash MG, Flores EY, et al. The Surface Protein SdrF Mediates Staphylococcus epidermidis Adherence to Keratin. J Infect Dis. 2017;215:1846-54. doi:10.1093/infdis/jix213.

14. Kogan G, Sadovskaya I, Chaignon P, Chokr A, Jabbouri S. Biofilms of clinical strains of Staphylococcus that do not contain polysaccharide intercellular adhesin. FEMS Microbiol Lett. 2006;255:11-6. doi:10.1111/j.1574-6968.2005.00043.x.

15. Rohde H, Burandt EC, Siemssen N, Frommelt L, Burdelski C, Wurster S, et al. Polysaccharide intercellular adhesin or protein factors in biofilm accumulation of Staphylococcus epidermidis and Staphylococcus aureus isolated from prosthetic hip and knee joint infections. Biomaterials. 2007;28:1711-20. doi:10.1016/j.biomaterials.2006.11.046. 
16. Pedroso SHSP, Sandes SHC, Luiz KCM, Dias RS, Filho RAT, Serufo JC, et al. Biofilm and toxin profile: A phenotypic and genotypic characterization of coagulase-negative staphylococci isolated from human bloodstream infections. Microb Pathog. 2016;100:312-8. doi:10.1016/j.micpath.2016.10.005.

17. Balaban N, Rasooly A. Staphylococcal enterotoxins. International Journal of Food Microbiology. 2000;61:1-10. doi:10.1016/S0168-1605(00)00377-9.

18. Boerema JA, Clemens R, Brightwell G. Evaluation of molecular methods to determine enterotoxigenic status and molecular genotype of bovine, ovine, human and food isolates of Staphylococcus aureus. Int J Food Microbiol. 2006;107:192-201. doi:10.1016/j.ijfoodmicro.2005.07.008.

19. Thomas DY, Jarraud S, Lemercier B, Cozon G, Echasserieau K, Etienne J, et al. Staphylococcal enterotoxin-like toxins $\mathrm{U} 2$ and $\mathrm{V}$, two new staphylococcal superantigens arising from recombination within the enterotoxin gene cluster. Infect Immun. 2006;74:4724-34. doi:10.1128/IAI.00132-06.

20. Chan WC, Coyle BJ, Williams P. Virulence regulation and quorum sensing in staphylococcal infections: Competitive AgrC antagonists as quorum sensing inhibitors. Journal of Medicinal Chemistry. 2004;47:4633-41. doi:10.1021/jm0400754.

21. Kong KF, Vuong C, Otto M. Staphylococcus quorum sensing in biofilm formation and infection. International Journal of Medical Microbiology. 2006;296:133-9. doi:10.1016/j.ijmm.2006.01.042.

22. Dufour $P$, Jarraud S, Vandenesch F, Greenland T, Novick RP, Bes M, et al. High genetic variability of the agr locus in Staphylococcus species. J Bacteriol. 2002;184:1180-6. doi:10.1128/jb.184.4.11801186.2002.

23. Becker K, Heilmann C, Peters G. Coagulase-negative staphylococci. Clin Microbiol Rev. 2014;27:870926. doi:10.1128/CMR.00109-13.

24. Turchi B, Bertelloni F, Marzoli F, Cerri D, Tola S, Azara E, et al. Coagulase negative staphylococci from ovine milk: Genotypic and phenotypic characterization of susceptibility to antibiotics, disinfectants and biofilm production. Small Rumin Res. 2020;183:106030. doi:10.1016/j.smallrumres.2019.106030.

25. Arciola CR, Campoccia D, Gamberini S, Cervellati M, Donati E, Montanaro L. Detection of slime production by means of an optimised Congo red agar plate test based on a colourimetric scale in Staphylococcus epidermidis clinical isolates genotyped for ica locus. Biomaterials. 2002;23:4233-9. doi:10.1016/S0142-9612(02)00171-0.

26. Frebourg NB, Lefebvre S, Baert S, Lemeland JF. PCR-based assay for discrimination between invasive and contaminating Staphylococcus epidermidis strains. J Clin Microbiol. 2000;38:877-80. doi:10.1128/jcm.38.2.877-880.2000.

27. Mekni MA, Bouchami O, Achour W, Ben Hassen A. Strong biofilm production but not adhesion virulence factors can discriminate between invasive and commensal Staphylococcus epidermidis strains. APMIS. 2012;120:605-11. doi:10.1111/j.1600-0463.2012.02877.x.

28. Abbondio M, Fois I, Longheu C, Azara E, Tola S. Biofilm production, quorum sensing system and analysis of virulence factors of Staphylococcus epidermidis collected from sheep milk samples. 
Small Rumin Res. 2019;174:83-7. doi:10.1016/j.smallrumres.2019.03.017.

29. Salgueiro VC, lorio NLP, Ferreira MC, Chamon RC, Dos Santos KRN. Methicillin resistance and virulence genes in invasive and nasal Staphylococcus epidermidis isolates from neonates. BMC Microbiol. 2017;17:15. doi:10.1186/s12866-017-0930-9.

30. Qin Z, Ou Y, Yang L, Zhu Y, Tolker-Nielsen T, Molin S, et al. Role of autolysin-mediated DNA release in biofilm formation of Staphylococcus epidermidis. Microbiology. 2007;153:2083-92. doi:10.1099/mic.0.2007/006031-0.

31. Christner M, Franke GC, Schommer NN, Wendt U, Wegert K, Pehle P, et al. The giant extracellular matrix-binding protein of Staphylococcus epidermidis mediates biofilm accumulation and attachment to fibronectin. Mol Microbiol. 2010;75:187-207. doi:10.1111/j.1365-2958.2009.06981.x.

32. Li M, Guan M, Jiang XF, Yuan FY, Xu M, Zhang WZ, et al. Genetic polymorphism of the accessory gene regulator (agr) locus in Staphylococcus epidermidis and its association with pathogenicity. J Med Microbiol. 2004;53:545-9. doi:10.1099/jmm.0.05406-0.

33. Fredheim EGA, Klingenberg C, Rohde H, Frankenberger S, Gaustad P, Flægstad T, et al. Biofilm formation by staphylococcus haemolyticus. J Clin Microbiol. 2009;47:1172-80. doi:10.1128/JCM.01891-08.

34. Otto M. Staphylococcus epidermidis - The "accidental" pathogen. Nature Reviews Microbiology. 2009;7:555-67. doi:10.1038/nrmicro2182.

35. Giormezis N, Kolonitsiou F, Foka A, Drougka E, Liakopoulos A, Makri A, et al. Coagulase-negative staphylococcal bloodstream and prosthetic-device-associated infections: The role of biofilm formation and distribution of adhesin and toxin genes. J Med Microbiol. 2014;63:1500-8. doi:10.1099/jmm.0.075259-0.

36. Da Cunha MDLRS, Calsolari RAO, Araújo JP. Detection of enterotoxin and toxic shock syndrome toxin 1 genes in Staphylococcus, with emphasis on coagulase-negative staphylococci. Microbiol Immunol. 2007;51:381-90. doi:10.1111/j.1348-0421.2007.tb03925.x.

37. Freeman DJ, Falkiner FR, Keane CT. New method for detecting slime production by coagulase negative staphylococci. J Clin Pathol. 1989;42:872-4. doi:10.1136/jcp.42.8.872.

38. Onni T, Sanna G, Larsen J, Tola S. Antimicrobial susceptibilities and population structure of Staphylococcus epidermidis associated with ovine mastitis. Vet Microbiol. 2011;148:45-50. doi:10.1016/j.vetmic.2010.07.024.

39. Lina G, Boutite F, Tristan A, Bes M, Etienne J, Vandenesch F. Bacterial competition for human nasal cavity colonization: Role of Staphylococcal agr alleles. Appl Environ Microbiol. 2003;69:18-23. doi:10.1128/AEM.69.1.18-23.2003.

40. Vandecasteele SJ, Peetermans WE, Merckx RR, Rijnders BJA, Van Eldere J. Reliability of the ica, aap and atlE genes in the discrimination between invasive, colonizing and contaminant Staphylococcus epidermidis isolates in the diagnosis of catheter-related infections. Clin Microbiol Infect. 2003;9:1149. doi:10.1046/j.1469-0691.2003.00544.x. 
41. Gu J, Li H, Li M, Vuong C, Otto M, Wen Y, et al. Bacterial insertion sequence IS256 as a potential molecular marker to discriminate invasive strains from commensal strains of Staphylococcus epidermidis. J Hosp Infect. 2005;61:342-8. doi:10.1016/j.jhin.2005.04.017.

42. Møretrø T, Hermansen L, Holck AL, Sidhu MS, Rudi K, Langsrud S. Biofilm formation and the presence of the intercellular adhesion locus ica among staphylococi from food and food processing environments. Appl Environ Microbiol. 2003;69:5648-55. doi:10.1128/AEM.69.9.5648-5655.2003.

43. Lou Q, Zhu T, Hu J, Ben H, Yang J, Yu F, et al. Role of the SaeRS two-component regulatory system in Staphylococcus epidermidis autolysis and biofilm formation. BMC Microbiol. 2011;11:146. doi:10.1186/1471-2180-11-146.

44. Monday SR, Bohach GA. Use of multiplex PCR to detect classical and newly described pyrogenic toxin genes in staphylococcal isolates. J Clin Microbiol. 1999;37:3411-4. doi:10.1128/jcm.37.10.3411-3414.1999.

45. Johnson WM, Tyler SD, Ewan EP, Ashton FE, Pollard DR, Rozee KR. Detection of genes for enterotoxins, exfoliative toxins, and toxic shock syndrome toxin 1 in Staphylococcus aureus by the polymerase chain reaction. J Clin Microbiol. 1991;29:426-30. doi:10.1128/jcm.29.3.426-430.1991.

46. Løvseth A, Loncarevic S, Berdal KG. Modified multiplex PCR method for detection of pyrogenic exotoxin genes in staphylococcal isolates. J Clin Microbiol. 2004;42:3869-72. doi:10.1128/JCM.42.8.3869-3872.2004.

\section{Tables}

Tables 1-3 are available in the Supplementary Files.

\section{Supplementary Files}

This is a list of supplementary files associated with this preprint. Click to download.

- Tables.docx 